\title{
Impact of the Assembly Method on the Surface Area-to-Volume Ratio and Electrochemical Oxidation Potential of Metal Nanospheres
}

\author{
Dhruba K. Pattadar, ${ }^{1}$ Jay N. Sharma, ${ }^{1}$ Badri P. Mainali and Francis P. Zamborini* \\ Department of Chemistry, University of Louisville, Louisville, Kentucky 40292, United States
}

\author{
${ }^{1}$ DKP and JNS contributed equally to this work. \\ *Corresponding Author
}

Email: f.zamborini@louisville.edu

\section{Supporting Information}



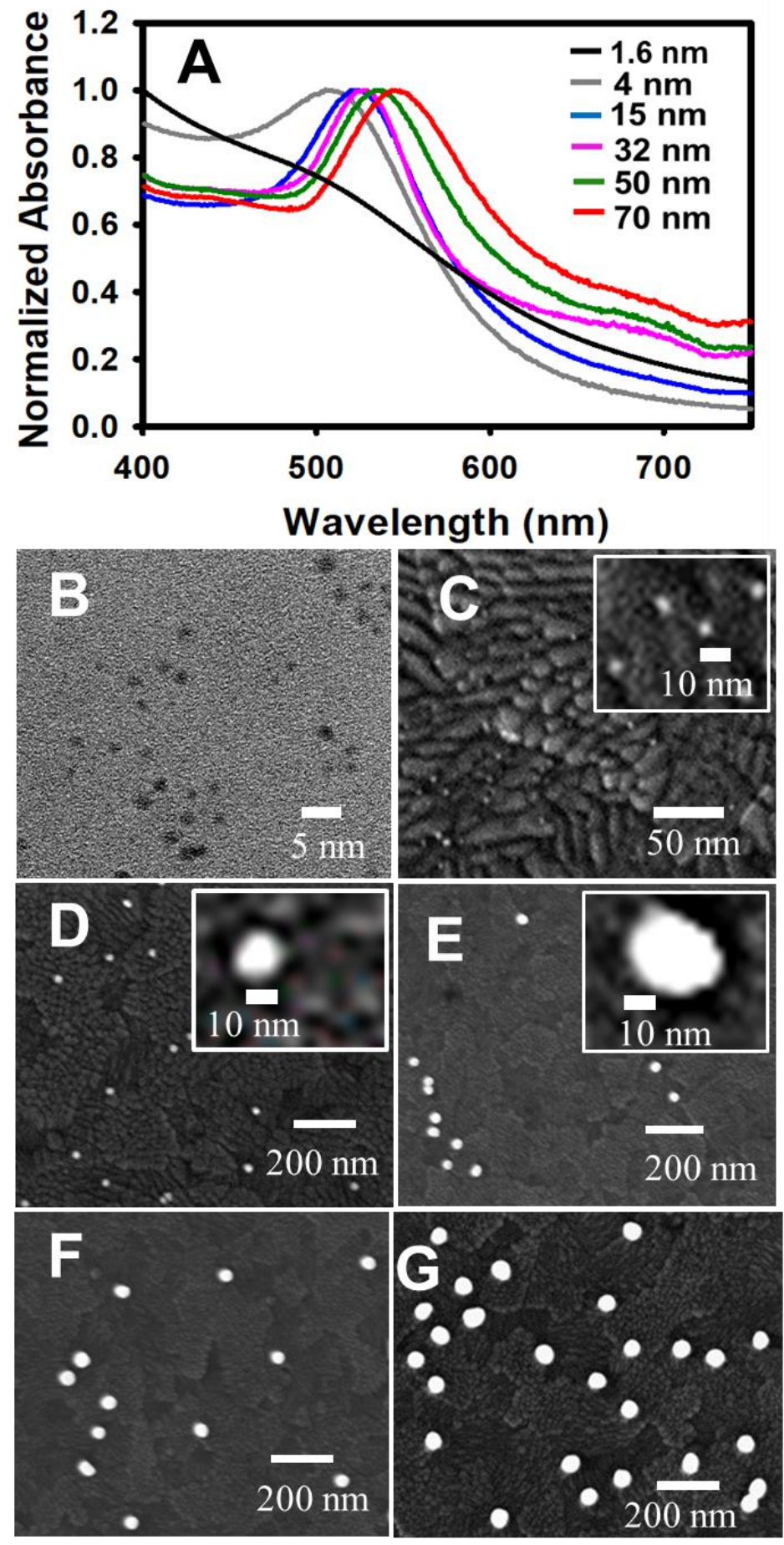

Figure S1. (A) UV-vis spectra of all Au nanospheres (NSs) in this study. (B) TEM image of $1.6 \pm 0.4 \mathrm{~nm}$ diameter THPC Au NSs. (C-G) SEM images of $4.1 \pm 0.7,15.1 \pm 1.8,31.2 \pm 1.9,50.1 \pm 3.0$, and $70.8 \pm 2.7 \mathrm{~nm}$ diameter citrate-coated Au NSs. Enlarged images of the Au NSs are shown in the insets. 

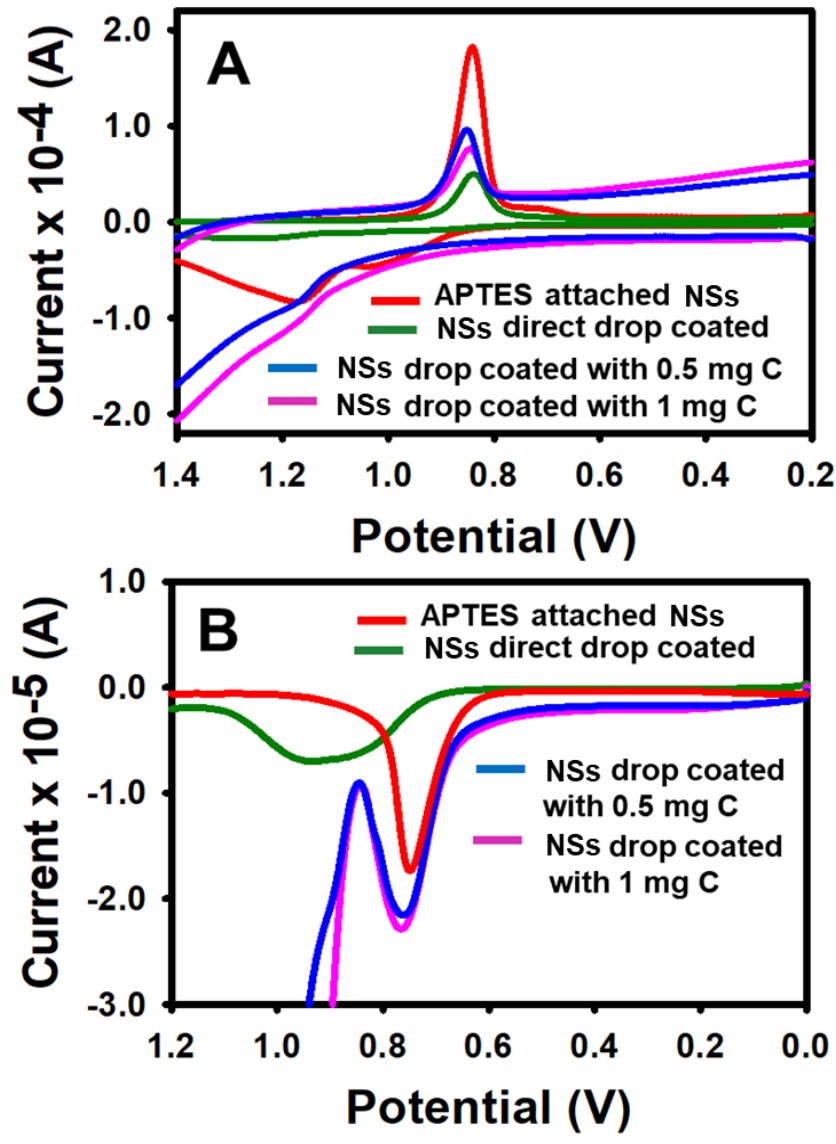

Figure S2. (A) CVs performed in $0.1 \mathrm{M} \mathrm{HClO}_{4}$ and (B) ASVs performed in $10 \mathrm{mM} \mathrm{KBr}+0.1 \mathrm{M}$ $\mathrm{KClO}_{4}$ of glass/ITO electrodes coated with $4 \mathrm{~nm}$ diameter Au NSs assembled by the different methods described. 
Table S1. Charge under the Au oxide reduction peak (from CVs), charge under the anodic stripping peaks (from ASVs), SA/V, average $\mathrm{SA} / \mathrm{V}$ ( \pm std. dev.), oxidation peak potentials $\left(\mathrm{E}_{\mathrm{p}, \mathrm{ox}}\right)$, and average $\mathrm{E}_{\mathrm{p}, \mathrm{ox}}( \pm$ std. dev.) as a function of NS size and assembly method (with ozone treatment for all sizes except $1.6 \mathrm{~nm}$ Au NSs).

\begin{tabular}{|c|c|c|c|c|c|c|}
\hline $\begin{array}{c}\text { Au NS } \\
\text { assembly } \\
\text { method }\end{array}$ & $\begin{array}{c}\text { CV } \\
\text { Measured } \\
\text { Surface Area } \\
\text { (Coulombs) }\end{array}$ & $\begin{array}{c}\text { ASV } \\
\text { Measured } \\
\text { Volume } \\
\text { (Coulombs) }\end{array}$ & $\begin{array}{c}\text { Surface } \\
\text { Area-to- } \\
\text { Volume } \\
\text { Ratio } \\
\text { (SA/V) }\end{array}$ & $\begin{array}{c}\text { Average } \\
\text { SA/V } \pm \\
\text { Std. Dev. }\end{array}$ & $\begin{array}{c}\text { Oxidation } \\
\text { peak } \\
\text { potential } \\
\left(\mathbf{E}_{\mathbf{p}, \mathbf{o x}}\right)\end{array}$ & $\begin{array}{c}\text { Average } \\
\text { E }_{\mathrm{p}, \mathrm{xx}} \pm \\
\text { Std. Dev. }\end{array}$ \\
\hline \multirow{3}{*}{$\begin{array}{c}4 \mathrm{~nm} \\
\text { APTES } \\
\text { (ozone) }\end{array}$} & $3.54 \times 10^{-5}$ & $3.76 \times 10^{-5}$ & 0.966 & \multirow[t]{3}{*}{$\begin{array}{c}0.948 \pm \\
0.019\end{array}$} & 0.711 & \multirow[t]{3}{*}{$\begin{array}{c}0.714 \pm \\
0.004\end{array}$} \\
\hline & $5.17 \times 10^{-5}$ & $5.42 \times 10^{-5}$ & 0.953 & & 0.719 & \\
\hline & $3.22 \times 10^{-5}$ & $3.47 \times 10^{-5}$ & 0.927 & & 0.713 & \\
\hline \multirow[t]{3}{*}{$\begin{array}{c}4 \text { nm EPD } \\
\text { (ozone) }\end{array}$} & $3.40 \times 10^{-5}$ & $4.41 \times 10^{-5}$ & 0.770 & \multirow[t]{3}{*}{$\begin{array}{c}0.801 \pm \\
0.030\end{array}$} & 0.728 & \multirow[t]{3}{*}{$\begin{array}{c}0.734 \pm \\
0.006\end{array}$} \\
\hline & $8.50 \times 10^{-5}$ & $1.02 \times 10^{-4}$ & 0.833 & & 0.741 & \\
\hline & $1.48 \times 10^{-5}$ & $1.86 \times 10^{-5}$ & 0.795 & & 0.732 & \\
\hline \multirow{3}{*}{$\begin{array}{c}15 \mathrm{~nm} \\
\text { APTES } \\
\text { (ozone) }\end{array}$} & $1.27 \times 10^{-5}$ & $3.31 \times 10^{-5}$ & 0.384 & \multirow[t]{3}{*}{$\begin{array}{c}0.370 \pm \\
0.022\end{array}$} & 0.740 & \multirow[t]{3}{*}{$\begin{array}{c}0.747 \pm \\
0.008\end{array}$} \\
\hline & $1.62 \times 10^{-5}$ & $4.23 \times 10^{-5}$ & 0.383 & & 0.757 & \\
\hline & $2.11 \times 10^{-5}$ & $6.12 \times 10^{-5}$ & 0.345 & & 0.746 & \\
\hline \multirow{3}{*}{$\begin{array}{c}15 \mathrm{~nm} \\
\text { EPD } \\
\text { (ozone) }\end{array}$} & $5.27 \times 10^{-5}$ & $1.90 \times 10^{-4}$ & 0.277 & \multirow[t]{3}{*}{$\begin{array}{c}0.278 \pm \\
0.017\end{array}$} & 0.780 & \multirow[t]{3}{*}{$\begin{array}{c}0.780 \pm \\
0.002\end{array}$} \\
\hline & $1.95 \times 10^{-5}$ & $7.47 \times 10^{-5}$ & 0.261 & & 0.783 & \\
\hline & $1.28 \times 10^{-5}$ & $4.31 \times 10^{-5}$ & 0.296 & & 0.779 & \\
\hline \multirow{3}{*}{$\begin{array}{c}32 \mathrm{~nm} \\
\text { APTES } \\
\text { (ozone) }\end{array}$} & $4.71 \times 10^{-6}$ & $2.30 \times 10^{-5}$ & 0.204 & \multirow[t]{3}{*}{$\begin{array}{c}0.201 \pm \\
0.002\end{array}$} & 0.795 & \multirow[t]{3}{*}{$\begin{array}{c}0.804 \pm \\
0.009\end{array}$} \\
\hline & $7.96 \times 10^{-6}$ & $3.99 \times 10^{-5}$ & 0.199 & & 0.805 & \\
\hline & $1.62 \times 10^{-5}$ & $8.02 \times 10^{-5}$ & 0.201 & & 0.813 & \\
\hline $\begin{array}{l}32 \mathrm{~nm} \\
\mathrm{EPD}\end{array}$ & $9.53 \times 10^{-6}$ & $1.22 \times 10^{-4}$ & 0.0781 & $\begin{array}{c}0.080 \pm \\
0.002\end{array}$ & 0.897 & $\begin{array}{c}0.898 \pm \\
0.012\end{array}$ \\
\hline
\end{tabular}




\begin{tabular}{|c|c|c|c|c|c|c|}
\hline \multirow[t]{2}{*}{ (ozone) } & $3.34 \times 10^{-6}$ & $4.02 \times 10^{-5}$ & 0.083 & & 0.911 & \\
\hline & $1.48 \times 10^{-6}$ & $1.87 \times 10^{-5}$ & 0.079 & & 0.887 & \\
\hline \multirow{3}{*}{$\begin{array}{c}50 \mathrm{~nm} \\
\text { APTES } \\
\text { (ozone) }\end{array}$} & $4.26 \times 10^{-6}$ & $2.74 \times 10^{-5}$ & 0.155 & \multirow[t]{3}{*}{$\begin{array}{c}0.123 \pm \\
0.027\end{array}$} & 0.898 & \multirow[t]{3}{*}{$\begin{array}{c}0.904 \pm \\
0.006\end{array}$} \\
\hline & $2.46 \times 10^{-6}$ & $2.15 \times 10^{-5}$ & 0.114 & & 0.906 & \\
\hline & $1.52 \times 10^{-5}$ & $1.49 \times 10^{-4}$ & 0.102 & & 0.910 & \\
\hline \multirow{3}{*}{$\begin{array}{c}50 \mathrm{~nm} \\
\text { EPD } \\
\text { (ozone) }\end{array}$} & $8.78 \times 10^{-7}$ & $1.71 \times 10^{-5}$ & 0.051 & \multirow[t]{3}{*}{$\begin{array}{c}0.055 \pm \\
0.004\end{array}$} & 0.940 & \multirow[t]{3}{*}{$\begin{array}{c}0.944 \pm \\
0.005\end{array}$} \\
\hline & $3.72 \times 10^{-6}$ & $6.31 \times 10^{-5}$ & 0.059 & & 0.942 & \\
\hline & $6.10 \times 10^{-6}$ & $1.10 \times 10^{-4}$ & 0.055 & & 0.951 & \\
\hline \multirow{3}{*}{$\begin{array}{c}70 \mathrm{~nm} \\
\text { APTES } \\
\text { (ozone) }\end{array}$} & $4.41 \times 10^{-5}$ & $8.19 \times 10^{-4}$ & 0.054 & \multirow[t]{3}{*}{$\begin{array}{c}0.073 \pm \\
0.017\end{array}$} & 0.911 & \multirow[t]{3}{*}{$\begin{array}{c}0.916 \pm \\
0.006\end{array}$} \\
\hline & $3.73 \times 10^{-6}$ & $4.27 \times 10^{-5}$ & 0.087 & & 0.915 & \\
\hline & $6.31 \times 10^{-5}$ & $8.10 \times 10^{-4}$ & 0.078 & & 0.923 & \\
\hline \multirow{3}{*}{$\begin{array}{c}70 \mathrm{~nm} \\
\text { EPD } \\
\text { (ozone) }\end{array}$} & $3.69 \times 10^{-6}$ & $1.51 \times 10^{-4}$ & 0.0244 & \multirow[t]{3}{*}{$\begin{array}{c}0.027 \pm \\
0.003\end{array}$} & 0.977 & \multirow[t]{3}{*}{$\begin{array}{c}0.982 \pm \\
0.008\end{array}$} \\
\hline & $2.28 \times 10^{-6}$ & $8.50 \times 10^{-5}$ & 0.0268 & & 0.993 & \\
\hline & $2.32 \times 10^{-6}$ & $7.89 \times 10^{-5}$ & 0.0294 & & 0.978 & \\
\hline \multirow{3}{*}{$\begin{array}{c}1.6 \mathrm{~nm} \\
\text { APTES } \\
\text { (no ozone) }\end{array}$} & $5.63 \times 10^{-4}$ & $2.45 \times 10^{-4}$ & 2.30 & \multirow{3}{*}{$\begin{array}{c}2.32 \pm \\
0.11\end{array}$} & 0.721 & \multirow{3}{*}{ N/A } \\
\hline & $1.98 \times 10^{-4}$ & $8.11 \times 10^{-5}$ & 2.44 & & 0.684 & \\
\hline & $5.94 \times 10^{-6}$ & $2.67 \times 10^{-6}$ & 2.22 & & 0.668 & \\
\hline \multirow{4}{*}{$\begin{array}{c}1.6 \mathrm{~nm} \\
\text { Direct } \\
\text { attachment } \\
\text { (no ozone) }\end{array}$} & $7.84 \times 10^{-6}$ & $5.51 \times 10^{-6}$ & 1.42 & \multirow{3}{*}{$\begin{array}{c}1.47 \pm \\
0.15\end{array}$} & 0.658 & \multirow{3}{*}{ N/A } \\
\hline & $1.50 \times 10^{-4}$ & $9.08 \times 10^{-5}$ & 1.65 & & 0.708 & \\
\hline & $1.50 \times 10^{-5}$ & $1.10 \times 10^{-5}$ & 1.36 & & 0.662 & \\
\hline & $5.51 \times 10^{-5}$ & $1.10 \times 10^{-4}$ & 0.551 & $\begin{array}{c}0.615 \pm \\
0.055\end{array}$ & 0.779 & $\begin{array}{c}0.769 \pm \\
0.008\end{array}$ \\
\hline
\end{tabular}




\begin{tabular}{|c|c|c|c|c|c|c|}
\hline \multirow{2}{*}{$\begin{array}{c}1.6 \mathrm{~nm} 5 \\
\mu \mathrm{L} \text { drop } \\
\text { cast } \\
\text { (no ozone) }\end{array}$} & $5.91 \times 10^{-5}$ & $9.10 \times 10^{-5}$ & 0.649 & & 0.762 & \\
\hline & $5.55 \times 10^{-5}$ & $8.49 \times 10^{-5}$ & 0.645 & & 0.768 & \\
\hline \multirow{3}{*}{$\begin{array}{c}1.6 \mathrm{~nm} 5 \\
\mu \mathrm{L}+ \\
0.0005 \mathrm{~g} \\
\text { CB drop } \\
\text { cast } \\
\text { (no ozone) }\end{array}$} & $6.21 \times 10^{-6}$ & $9.62 \times 10^{-6}$ & 0.671 & \multirow[t]{3}{*}{$\begin{array}{c}0.661 \pm \\
0.090\end{array}$} & 0.772 & \multirow[t]{3}{*}{$\begin{array}{c}0.770 \pm \\
0.016\end{array}$} \\
\hline & $6.12 \times 10^{-6}$ & $8.20 \times 10^{-6}$ & 0.746 & & 0.754 & \\
\hline & $2.89 \times 10^{-5}$ & $5.10 \times 10^{-5}$ & 0.566 & & 0.786 & \\
\hline \multirow{3}{*}{$\begin{array}{c}1.6 \mathrm{~nm} 5 \\
\mu \mathrm{L}+0.001 \\
\mathrm{~g} \mathrm{CB} \text { drop } \\
\text { cast } \\
\text { (no ozone) }\end{array}$} & $2.21 \times 10^{-5}$ & $5.27 \times 10^{-5}$ & 0.419 & \multirow[t]{3}{*}{$\begin{array}{c}0.354 \pm \\
0.058\end{array}$} & 0.791 & \multirow[t]{3}{*}{$\begin{array}{c}0.791 \pm \\
0.002\end{array}$} \\
\hline & $4.89 \times 10^{-5}$ & $1.60 \times 10^{-4}$ & 0.306 & & 0.790 & \\
\hline & $2.74 \times 10^{-5}$ & $8.10 \times 10^{-5}$ & 0.338 & & 0.794 & \\
\hline \multirow{3}{*}{$\begin{array}{c}1.6 \mathrm{~nm} 5 \\
\mu \mathrm{L}+0.004 \\
\mathrm{~g} \mathrm{CB} \text { drop } \\
\text { cast } \\
\text { (no ozone) }\end{array}$} & $4.46 \times 10^{-5}$ & $1.98 \times 10^{-4}$ & 0.225 & \multirow[t]{3}{*}{$\begin{array}{c}0.207 \pm \\
0.028\end{array}$} & 0.827 & \multirow[t]{3}{*}{$\begin{array}{c}0.841 \pm \\
0.012\end{array}$} \\
\hline & $6.35 \times 10^{-5}$ & $2.85 \times 10^{-4}$ & 0.222 & & 0.849 & \\
\hline & $7.45 \times 10^{-5}$ & $4.25 \times 10^{-4}$ & 0.175 & & 0.847 & \\
\hline \multirow{3}{*}{$\begin{array}{c}4 \mathrm{~nm} 5 \mu \mathrm{L} \\
\text { drop cast } \\
\text { (ozone) }\end{array}$} & $1.29 \times 10^{-5}$ & $4.54 \times 10^{-5}$ & 0.284 & \multirow[t]{3}{*}{$\begin{array}{c}0.261 \pm \\
0.036\end{array}$} & 0.858 & \multirow[t]{3}{*}{$\begin{array}{c}0.865 \pm \\
0.007\end{array}$} \\
\hline & $7.88 \times 10^{-6}$ & $3.59 \times 10^{-5}$ & 0.219 & & 0.873 & \\
\hline & $2.29 \times 10^{-5}$ & $8.14 \times 10^{-5}$ & 0.281 & & 0.865 & \\
\hline \multirow{3}{*}{$\begin{array}{c}4 \mathrm{~nm} 5 \mu \mathrm{L} \\
+0.0005 \mathrm{~g} \\
\text { CB drop } \\
\text { cast } \\
\text { (ozone) }\end{array}$} & $3.84 \times 10^{-5}$ & $5.41 \times 10^{-5}$ & 0.709 & \multirow[t]{3}{*}{$\begin{array}{c}0.669 \pm \\
0.006\end{array}$} & 0.758 & \multirow[t]{3}{*}{$\begin{array}{c}0.755 \pm \\
0.005\end{array}$} \\
\hline & $3.15 \times 10^{-5}$ & $5.31 \times 10^{-5}$ & 0.593 & & 0.759 & \\
\hline & $9.62 \times 10^{-6}$ & $1.36 \times 10^{-5}$ & 0.707 & & 0.749 & \\
\hline \multirow{3}{*}{$\begin{array}{c}4 \mathrm{~nm} 5 \mu \mathrm{L} \\
+0.001 \mathrm{~g} \\
\mathrm{CB} \text { drop } \\
\text { cast } \\
\text { (ozone) }\end{array}$} & $2.61 \times 10^{-5}$ & $5.87 \times 10^{-5}$ & 0.444 & \multirow[t]{3}{*}{$\begin{array}{c}0.474 \pm \\
0.028\end{array}$} & 0.782 & \multirow[t]{3}{*}{$\begin{array}{c}0.785 \pm \\
0.007\end{array}$} \\
\hline & $2.73 \times 10^{-5}$ & $5.71 \times 10^{-5}$ & 0.478 & & 0.793 & \\
\hline & $1.83 \times 10^{-5}$ & $3.66 \times 10^{-5}$ & 0.500 & & 0.780 & \\
\hline $\begin{array}{l}4 \mathrm{~nm} 5 \mu \mathrm{L} \\
+0.004 \mathrm{~g}\end{array}$ & $1.88 \times 10^{-5}$ & $7.10 \times 10^{-5}$ & 0.265 & $\begin{array}{c}0.306 \pm \\
0.035\end{array}$ & 0.806 & $\begin{array}{c}0.809 \pm \\
0.005\end{array}$ \\
\hline
\end{tabular}




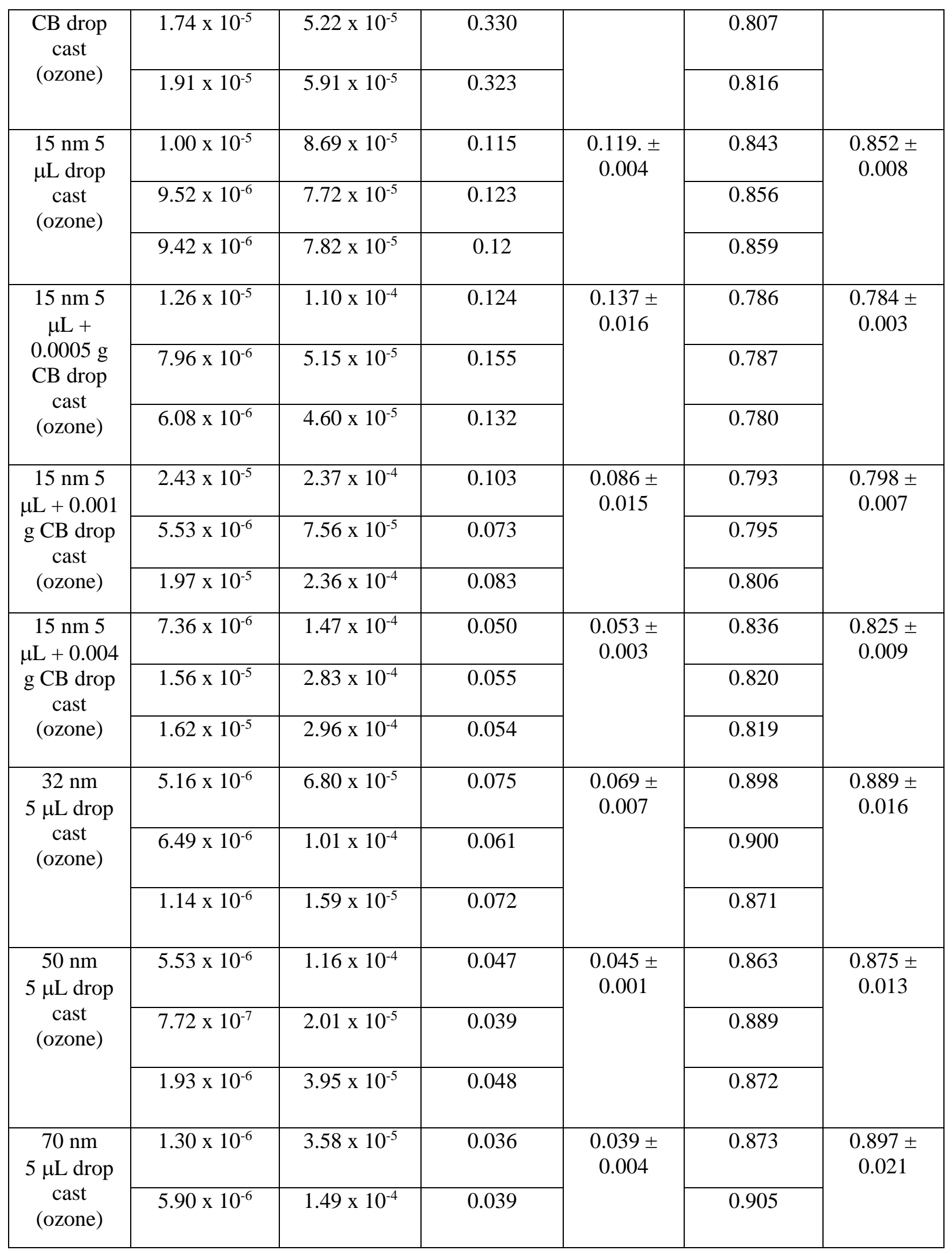




\begin{tabular}{|l|l|l|l|l|l|l|}
\hline & $4.76 \times 10^{-6}$ & $1.08 \times 10^{-4}$ & 0.044 & & 0.914 & \\
\hline
\end{tabular}

Table S2. Charge under the Au oxide reduction peak (from CVs), charge under the anodic stripping peak (from ASVs), peak oxidation potential $\left(\mathrm{E}_{\mathrm{p}, \mathrm{ox}}\right)$, and average $\mathrm{E}_{\mathrm{p}, \mathrm{ox}}( \pm \mathrm{std}$. dev.) for 1.6 $\mathrm{nm}$ diameter Au NSs assembled by APTES linking and direct soaking.

\begin{tabular}{|c|c|c|c|}
\hline Assembly method & $\begin{array}{c}\text { Charge from ASV } \\
\text { (Coulombs) } \\
\text { "Volume of Au } \\
\text { NSs" } \\
\end{array}$ & $\begin{array}{l}\text { Oxidation peak } \\
\text { potential }\left(\mathbf{E}_{\mathbf{p}, \mathbf{o x}}\right)\end{array}$ & $\begin{array}{l}\text { Average } E_{p, o x} \pm \\
\text { Std. Dev. }\end{array}$ \\
\hline \multirow{3}{*}{$\begin{array}{c}\text { Glass ITO/APTES/1.6 nm } \\
\text { Au NSs }\end{array}$} & $8.79 \times 10^{-5}$ & 0.466 & \multirow{3}{*}{$0.456 \pm 0.008$} \\
\hline & $5.01 \times 10^{-5}$ & 0.452 & \\
\hline & $2.76 \times 10^{-5}$ & 0.451 & \\
\hline \multirow{3}{*}{$\begin{array}{c}\text { Glass ITO/1.6 nm Au NSs } \\
\text { (Direct attachment) }\end{array}$} & $1.74 \times 10^{-5}$ & 0.512 & \multirow{3}{*}{$0.504 \pm 0.015$} \\
\hline & $5.42 \times 10^{-5}$ & 0.515 & \\
\hline & $1.07 \times 10^{-5}$ & 0.487 & \\
\hline
\end{tabular}



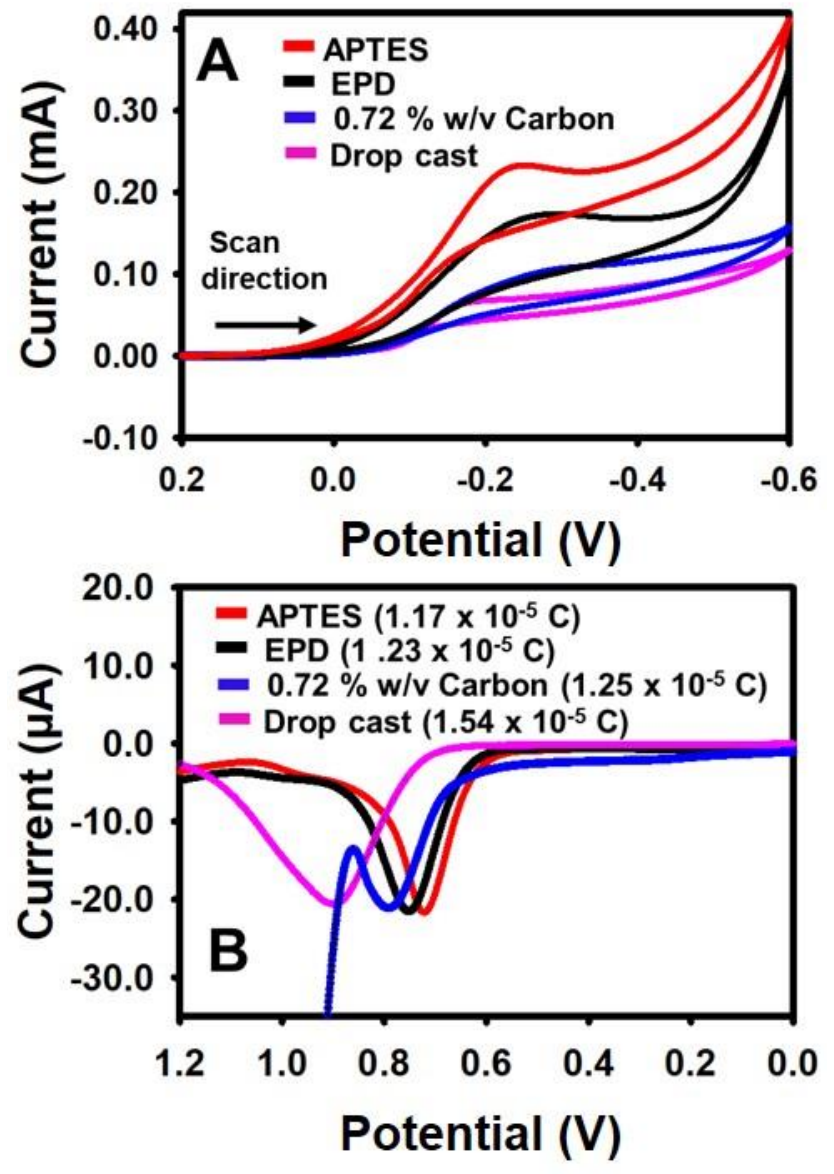

Figure S3. (A) ORR for $4 \mathrm{~nm}$ diameter Au NSs assembled onto glass/ITO by APTES linker, EPD and drop cast deposition with and without carbon black. (B) ASVs of the different Au NSs on glass/ITO in (A), showing similar Au coverages for all of the assembly methods. 